\section{Molluscicidal Activity of Surface-active Agents on the Snail Biomphalaria sudanica; a Vector of Bilharzia}

Previous work on a naturally occurring substance which inhibits growth in Biomphalaria sudanica ${ }^{1}$ indicated that its toxicity was partly due to its surface activity. It was therefore decided to test various commercially produced surface-active agents, at various concentrations, on the survival of this species of snail.

As the result of requests to British manufacturers of surface-active agents for samples of their products, we obtained more than 100 compounds, which were dissolved at 5 different concentrations, ranging from $0 \cdot 1$ to 1,000 p.p.m., in an artificial medium containing the major inorganic ions found in Lake Victoria, in the concentrations in which they occur in the lake.

The surface tension of these solutions was determined at $30^{\circ} \mathrm{C}$ by means of the drop-weight method of Harkins and Brown $^{2}$, and the apparatus used was virtually identical with that described by Harkins and Alexander ${ }^{3}$.

For the 101 detergents tested it was found that 5 were non-toxic to the snails, even at concentrations of 1,000 p.p.m., that 22 were toxic at 1,000 p.p.m., 39 at 100 p.p.m., 23 at 10 p.p.m., 10 at 1 p.p.m., and, finally, 2 were found to kill the snails at a concentration as low as $0 \cdot 1$ p.p.m. Death of the snail was sometimes obviously due to hremolysis, but this effect did not occur each time, and the amount of hæmolysis was certainly unrelated to the toxicity of the surface-active components.

The substances which killed the snails at the lowest concentration so far investigated $(0 \cdot 1$ p.p.m.) were the tertiary amines 'Empigen $A E 3$ ' and 'Empigen $A N$ ' (the surface tensions measured at this concentration were respectively 71 and 65 dynes $\mathrm{cm}^{-1}$ ).

No direct correlation could be detected between the surface activity of the detergents and their toxicity on Biomphalaria. 'Ethylan $P C$ ', for example, had a surface tension of approximately 37 dynes $\mathrm{cm}^{-1}$ at a concentration of 1,000 p.p.m., but this proved, at this concentration, to be non-toxic to the snail. On the other hand, the most surface-active substance tested so far, 'Deciquam 222' (which at a concentration of 1,000 p.p.m. gave a surface tension of approximately 13 dynes $\mathrm{cm}^{-1}$ ), killed the snail at a concentration of 1 p.p.m. and a surface tension of 69 dynes $\mathrm{cm}^{-1}$.

It was found that the cationic detergents were, on the whole, more toxic than the anionic or non-ionic ones.

Mixtures of anionic and cationic agents were found, on the whole, to be less toxic then the surface-active agents separately. Thus, a mixture containing 100 p.p.m. 'Perlankrol $T L$ ' and 100 p.p.m. 'Cibaphasol $C$ ' did not kill snails even after $89 \mathrm{~h}$ whereas the separate components, at the same concentration, killed them after 20 and $5 \mathrm{~h}$ respectively. The addition of 'Silicone $M S$ antifoam $A^{\prime}$ ' (Hopkin and Williams) to the surface-active agents lowered the toxic concentration in most cases by a factor of $10-100$. The influence of the silicone was particularly evident in the case of the anionic and cationic agents. From these experiments it can be concluded that the toxic effect of most surface-active agents on Biomphalaria has both a chemical and a physical origin.

It was found that the eggs of Biomphalaria sudanica were more resistant to the surface-active compounds than was the snail itself. While the snails were killed within $1 \mathrm{~h}$ in certain detergents at a concentration of 1,000 p.p.m., most of the embryos in the eggs survived this concentration for more than $24 \mathrm{~h}$. Concentrations of 10 p.p.m. and less of any of the surface-active agents investigated had no detectable harmful effect on the embryos.

These findings may be useful in the selection of surfaceactive agents which, apart from their own molluscicidal activity, can also be used for the dispersion of almost insoluble molluscicides.

Department of Zoology,

S. A. VISSER

Makerere University College, Kampala, Uganda.

Berrie, A. D., and Visser, S. A., Physiol. Zool., 36, 167 (1963).

${ }^{2}$ Harkins, W. D., and Brown, F. E., J. Amer. Chem. Soc., 38, 248 (1916); 41, 499 (1919).

${ }^{3}$ Harkins, W. D., and Alexander, A. E., in Techniques of Organic Chemistry, edit. by Weissberger, A., 1, 776 (Interscience Publishers Inc., New York, 1959).

\section{Some Biological and Biochemical Properties of the Polarizing Factor in Hydra}

TRIPY: proposed a growth pattern for Hydra which was later confirmed by Brien ${ }^{2}$ and Burnett and Garofalo ${ }^{3}$. Beneath the hypostome of Hydra a growth region exists where cell proliferations constantly occur. Cell divisions in this area force adjacent cells distally to the tentacle tips and proximally to the basal disk. When cells reach the extremity of the body column they die and are sloughed off. Thus, the animal is capable of continual growth without a change in form.

Burnett ${ }^{4}$ postulated the existence of a growth-stimulating principle which controls cell division and ultimately head formation along the length of the body column. According to this hypothesis, the growth-stimulating material is concentrated in the hypostome. It diffuses proximally from this location in a gradient until it reaches the basal disk. The reason that heads fail to form along the entire column is becauso of the presence of a postulated inhibitor, produced in the sub-hypostomal growth region which also diffuses proximally. This inhibitor specifically blocks the action of the stimulator and the ratio of stimulator to inhibitor in any body region determines the form of that region. For example, in the budding region the inhibitor is dilute and the residual stimulator in this region induces cell divisions necessary for the elaboration of a new polyp. If the growth pattern in Hydra is chemically controlled as hypothesized, it should be possible to define quantitatively the mode of action of these agents and to purify them.

This communication reports preliminary investigation into the chemical and biological nature of the growth stimulatory material only. We have isolated a substance from Hydra capable of inverting polarity. The material is obtained from homogenates of Hydra boiled $10 \mathrm{~min}$ to stimulate nematocyst discharge. (Nematocyst toxin is lethal to the Hydra after its release from the capsule.) The active factor is located in the supernatant of a fraction sedimented after centrifuging the homogenate at $25,000 \mathrm{~g}$ for $50 \mathrm{~min}$. Annuli of tissue excised from the gastricbudding region junction of $H y d r a$ pirardi when exposed to these extracts for $4 \mathrm{~h}$ exhibit subsequent excessive tentacle or head growth during regeneration. Inverted polarity develops in more than 20 per cent $(0.222)$ of the surviving animals as opposed to spontaneous production of supernumerary tentacles in less than 2 per cent $(0.016)$ of 387 control animals tested (that is, annuli allowed to regenerate in culture solution without prior incubation in an extract containing growth-stimulating principle). No control animal ever developed an excessive number of tentacles, only their placement along the body column was abnormal, and here never more than two tentacles were located outside the oral ring.

Growth-stimulating material is concentrated in the distal portion of normal animals (Table 1). The activity of this area approximates that of whole animal extracts. Although the mechanism of action of the extracted material is unknown, it probably enters the assay animal by diffusion during the incubation period. Evidence for this conjecture is, first, the material can be collected in agar blocks and can be introduced afterwards from the 